independent of glycaemic control. Thromb Haemost 72: 979-984

3. Gearing AJH, Hemingway I, Pigott R et al. (1992) Soluble forms of vascular adhesion molecules, E-selectin, ICAM-1 and VCAM-1: pathological significance. Annals NY Acad Sci 667: 324-331

4. Lampeter ER, Kishimoto TK, Rothlein R et al. (1992) Elevated levels of circulating adhesion molecules in IDDM patients and in subjects at risk for IDDM. Diabetes 41: 1668-1671

5. Blann AD, Tse W, Maxwell SJR, Waite MA (1994) Increased levels of the soluble adhesion molecule E-selectin in essential hypertension. J Hypertension 12: 925-928

6. Brownlee M, Cerami A, Vlassara H (1988) Advanced glycosylation end products in tissue and the biochemical basis of diabetic complications. N Engl J Med 318: 1315-1321

\section{Increased aortic stiffness in women with NIDDM}

\section{Dear Sir,}

Rydén Ahlgren et al. [1] report increased arterial stiffness in women with insulin-dependent diabetes mellitus (IDDM). In the Discussion section they state that "when distensibility is expressed as stiffness $(\beta)$, the dependence on pressure in the physiological range is largely abolished". The $\beta$ index is given by the expression $\ln \left(\mathrm{P}_{\mathrm{s}} / \mathrm{P}_{\mathrm{d}}\right) / \Delta \mathrm{D} / \mathrm{D}_{\mathrm{d}}$ where $\mathrm{P}_{\mathrm{s}}$ and $\mathrm{P}_{\mathrm{d}}$ are systolic and diastolic blood pressures (BP), and $\Delta \mathrm{D}$ is the change in diastolic lumen diameter, $D_{d}$, during the cardiac cycle. It is our experience that while this expression provides a useful BP correction in normal, healthy (particularly young) adult subjects [2], in older subjects or patients with vascular disease a considerable BP dependence can be observed $[3,4]$. We have a longstanding interest in studying aortic biophysical properties in vivo, based on aortic pulse wave velocity (PWV) measurements [2-4]. We have shown previously that adult patients with non-insulin-dependent diabetes (NIDDM) have significantly stiffer aortas than age- and sex-matched control subjects [2] and that young, normal healthy adult subjects with a positive family history of NIDDM have stiffer aortas than their age- and sex-matched counterparts with no family history of diabetes [5].

The paper by Rydén Ahlgren et al. [1] prompted us to reanalyse data from 48 (23 male) patients with NIDDM. The age of the subjects was $62.3 \pm 7.9$ years with mean BPs of $105 \pm 14 \mathrm{mmHg}$. No significant differences were observed between males and females in terms of age, BP, or body mass indices. Aortic PWV was measured along the descending thoraco-abdominal aorta as previously described [2-4], and the $\beta$ index was calculated as the inverse of a BP adjusted index of aortic distensibility [2]. Although there was a considerable overlap, females with NIDDM had significantly stiffer aortas compared with their male counterparts (females vs males, unpaired $t$-tests $-\mathrm{PWV}: 13.0 \pm 3.5$ vs $10.5 \pm 2.6 \mathrm{~m} / \mathrm{s}, p=0.01 ; \beta$ : $23.0 \pm 10.5$ vs $15.8 \pm 7.5$ (dimensionless) $p=0.009$ ). This observation may explain why diabetes is a much stronger risk factor for coronary heart disease in women than in men, and may be especially relevant to the general population as NIDDM is four times more prevalent than IDDM. Comparison of values from female NIDDM patients with values that we have observed in non-diabetic normal female subjects of similar ages [4] also revealed stiffer aortas in the NIDDM cohort [NIDDM vs control subjects - PWV: $13.0 \pm 3.5$ vs $9.1 \pm 1.7 \mathrm{~m} / \mathrm{s} ; \beta$ : $23.0 \pm 10.5$ vs $12.4 \pm 3.7$ (dimensionless)], results which support

Corresponding author: Dr. E. D. Lehmann, Academic Department of Radiology, St.Bartholomew's Hospital, London EC1A 7BE, UK

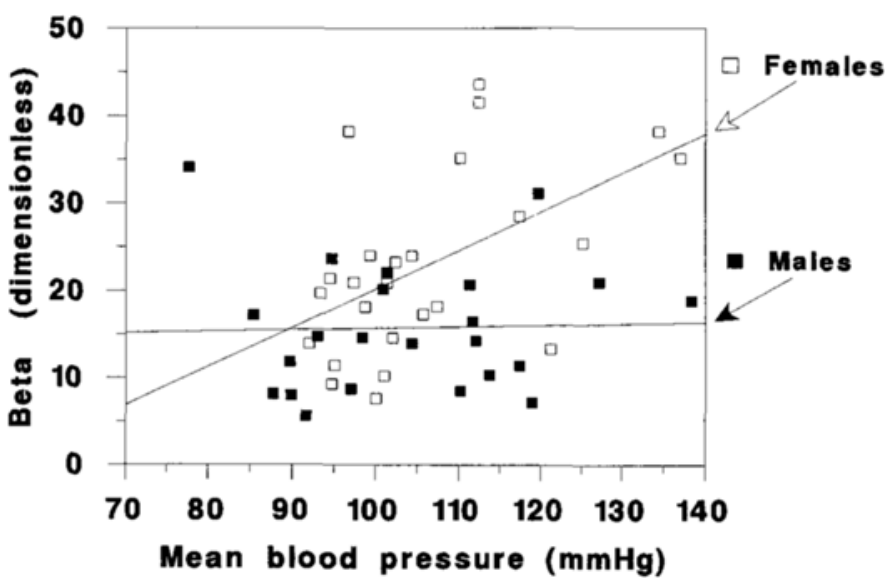

Fig. 1. Relation between mean blood pressure $(\mathrm{mmHg})$ and $\beta$ index in 48 (23 male) patients with NIDDM. Regression equations: females: $y=0.443 x-24.1(r=0.52, p=0.007)$; males: $y=0.017 x+14.0(r=0.03, p=0.88$, N.S. $)$

our previous observations in a smaller cohort of patients with NIDDM [2].

Figure 1 demonstrates the relation observed between $\beta$ and mean BP for male and female subjects separately. As can be seen while $\beta$ did not exhibit any correlation with pressure in the male cohort $(r=0.03, p=0.88, \mathrm{~N} . \mathrm{S}$. $)$, a significant relation was observed with $\mathrm{BP}$ in the female cohort $(r=0.52$, $p=0.007)$. While our patients are older and have NIDDM and many had symptomatic vascular disease as would be expected in a cohort of this age - we can only speculate whether similar relations were observed in the study of asymptomatic IDDM patients by Rydén Ahlgren et al. [1]. Would it be possible for the authors to look at the correlation coefficients, $p$ values, and regression equations observed in their study between $\beta$ and systolic, diastolic and mean BP separately for male and female IDDM patients? Our data, and the results of previous studies in non-diabetic subjects $[3,4]$, lead us to suspect that a differential correlation between $\beta$ and BP in males and females may well exist in the diabetic patients in their dataset. We would like to stress that we are not questioning the findings of Rydén Ahlgren et al. [1] that females with IDDM have stiffer aortas than males. We have made similar observations in patients with NIDDM. However, we do question the utility of applying the "blood pressure corrected" index, $\beta$, especially if the BP correction does not work fully, and appears to have differential effects between the cohorts of interest. Furthermore it may be methodologically more sound to provide the basic 'raw' distensibility data $\left(\Delta \mathrm{D} / \mathrm{D}_{\mathrm{d}}\right)$ as this was what was actually measured by their echo-tracking device - rather than attempting to apply a correction for BP - especially if on the basis of the above data and previously published reports $[3,4]$ the $\beta$-index does not appear to be completely 
reliable. In this respect we should make clear that our data comparing males and females are presented using the $\beta$ index simply to permit a comparison with the results of Rydén Ahlgren et al.

Finally, in the Discussion section the authors [1] referring to differences between central aortic and peripheral BP write "no significant age or gender-related differences are seen thus allowing comparisons between individuals and groups". Surely part of the issue here is whether the differences between intra-aortic BP and brachial BP change with diabetes status? We are not aware of any work comparing such measurements in diabetic and non-diabetic individuals. Thus, we have another variable which may be different between the control and diabetic cohorts studied and which needs to be considered. Given this, we simply do not know whether the authors' assumption that sphygmomanometrically measured BP in the brachial artery can be reliably substituted for the aortic BP at a single aortic cross-section in both diabetic and non-diabetic subjects is valid. To us this appears to be another compelling reason to report the 'raw', measured distensibility data $\left(\Delta \mathrm{D} / \mathrm{D}_{\mathrm{d}}\right)$ rather than a derived parameter such as $\beta$.
Yours sincerely,

E.D. Lehmann, K.D. Hopkins, R. G. Gosling

\section{References}

1. Rydén Ahlgren Å, Lanne T, Wollmer P, Sonesson B, Hansen $F$, Sundkvist $G$ (1995) Increased arterial stiffness in women, but not men, with IDDM. Diabetologia 38: 1082-1089

2. Lehmann ED, Hopkins KD, Gosling RG (1993) Aortic compliance measurements using Doppler ultrasound: in vivo biochemical correlates. Ultrasound Med Biol 19: 683-710

3. Lehmann ED, Hopkins KD, Parker JR et al. (1994) Aortic distensibility in post-menopausal women receiving $\mathrm{T}_{\mathrm{i}}^{*}$ bolone. Br J Radiol 67: 701-705

4. Lehmann ED, Hopkins KD, Jones RL, Rudd AG, Gosling RG (1995) Aortic distensibility in patients with cerebrovascular disease. Clin Sci 89: 247-253

5. Hopkins KD, Lehmann ED, Jones RL, Turay RC, Gosling RG (1996) A family history of NIDDM is associated with decreased aortic distensibility in normal healthy young adult subjects. Diabetes Care (in press)

\section{Increased arterial stiffness in IDDM women - methodological considerations}

Dear Sir,

We are pleased to learn of the interest Dr. Lehman et al. show in our recent paper.

In this paper stiffness $(\beta)$ data were presented as an expression of arterial distensibility. As suggested by Dr. Lehman et al. also the strain (fractional diameter change) = systolic diameter - diastolic diameter

$$
\text { diastolic diameter of the abdominal aorta }
$$
now is shown (Fig. 1). As can be seen, the aortic strain is significantly lower in women $(p=0.0025)$, but not in men $(p=0.9)$ with insulin-dependent diabetes mellitus (IDDM), as compared with healthy control subjects of corresponding age and sex, confirming the earlier presented $\beta$ results. However, strain is not only dependent on the vessel wall constituents but also on the distending force, that is, the blood pressure. The pressure/diameter relationship of the aorta is curvilinear as is evident from simultaneous recordings of arterial pressure and diameter with a more distensible part at lower than at higher pressures. Although there is no sharp inflection point in the curve, the transition from distensible to stiff behaviour occurs above $80 \mathrm{~mm} \mathrm{Hg}$ [1]. These mechanical characteristics have been attributed to the properties of the matrix in the wall, mainly elastin and collagen. The more distensible elastin is the principle load bearer at low pressures and small distensions, whereas both elastin and the stiffer collagen are load-bearing at high pressures and large distensions [2]. Thus, changes in mean distending pressure (MAP) and pulse pressure affect the aortic distensibility as well as the pulse wave velocity (PWV). In Figure 1 it can be seen that the strain in healthy control subjects decreases with age. However, MAP as well as pulse pressure
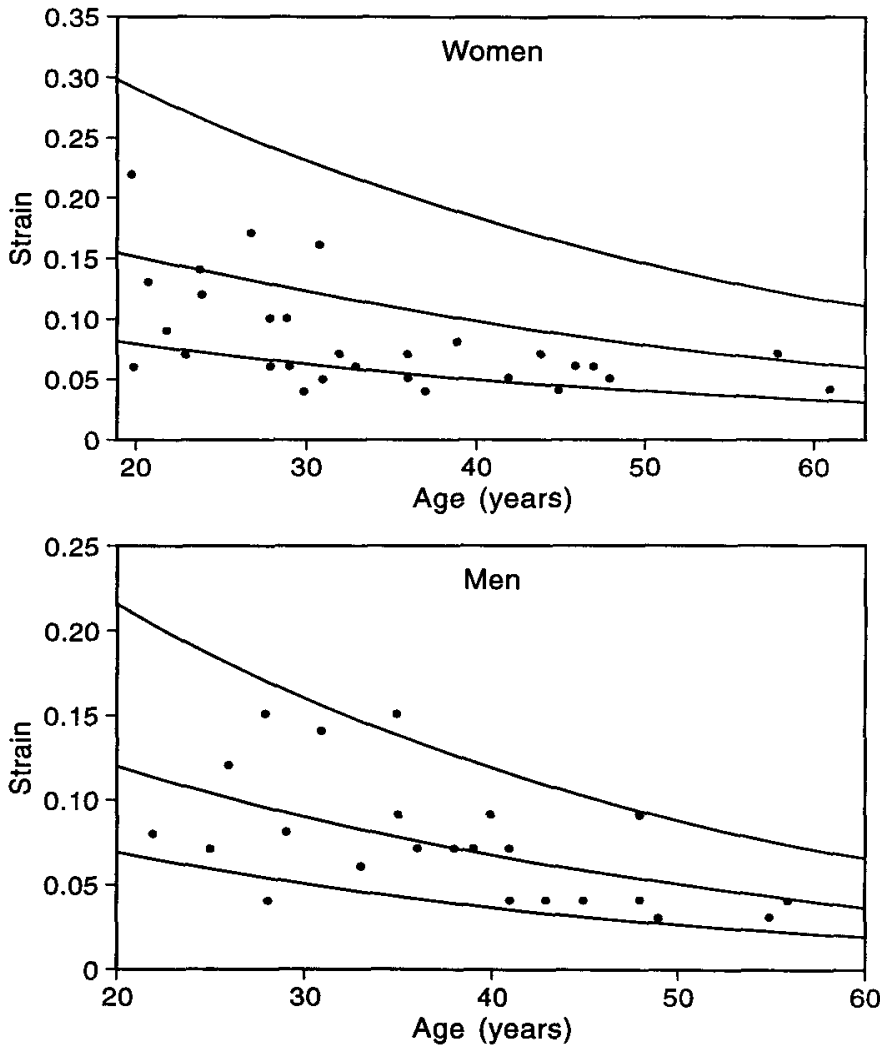

Fig. 1. Aortic strain (fractional diameter change) in women and men with IDDM. The lines represent mean, upper and lower of $95 \% \mathrm{CI}$, for the control women and men. Note the significantly decreased strain in women, but not in men with IDDM ( $p=0.0025$ and $p=0.9$, respectively)

Corresponding author: T.Länne, $\mathrm{MD}, \mathrm{PhD}$, Department of Vascular and Renal Diseases, Lund University, Malmö University Hospital, S-205 02 Malmö, Sweden 